\title{
Ideologías lingüísticas en el estudio de la 'competencia en lengua índigena' en chile. Implicancias teóricas y metodológicas
}

\section{Language Ideologies in the Study of 'Indigenous Language Competence' in Chile. Theoretical and Methodological Implications}

\begin{abstract}
MARCO ESPINOZA A.
Universidad de Chile, Departamento de Lingüística, Facultad de Filosofía y Humanidades. Chile. Correo electrónico: marcespi@uchile.cl
\end{abstract}

Mediante una discusión articulada en torno al concepto de ideología lingüística, en este trabajo se examinan críticamente aquellos estudios que han investigado la competencia en lengua indígena en Chile. Si bien dichos estudios no definen adecuadamente su objeto de análisis, todos ellos reflejan, tanto a nivel teórico como metodológico, la influencia de las ideologías monoglósica y de la lengua estándar. Estas ideologías lingüísticas imponen al estudio de dicha competencia en lengua indígena un sesgo monolingüe y una visión de las lenguas como entidades con una forma canónica uniforme. Se argumenta que las proposiciones contenidas en los estudios de competencia no solo no se corresponden con las características sociolingüísticas presentes en situaciones de contacto, sino que también repercuten en la evaluación que se hace de los sujetos al crear e imponer externamente jerarquías y categorías de hablantes. El artículo finaliza sugiriendo una aproximación diferente al estudio de la situación sociolingüística de las lenguas indígenas en Chile que supere la fijación con una noción de competencia vagamente definida y que invite al descubrimiento, la descripción y la comprensión de las prácticas lingüísticas y los repertorios verbales y comunicativos de -y desdelos hablantes, en relación directa con sus complejas y cambiantes realidades sociales, culturales y comunicativas.

Palabras clave: ideología lingüística, competencia en lengua indígena, hablantes en lengua indígena, sesgo monolingüe, lengua estándar.

This paper critically examines studies dealing with the so-called competence in an indigenous language in Chile. Through an analysis articulated around the notion of language ideology, it is shown that these studies are shaped, theoretically and methodologically, by the influence of monoglossic and standard language ideologies. These ideologies impose a monolingual bias and a view of languages as reified, standard and homogenous on the study of competence. It is argued 
that the propositions contained in these studies do not reflect the sociolinguistic characteristics of contact situations in Chile and that they enforce external categories and hierarchies of speakers. It is concluded by suggesting a different approach to the study of the sociolinguistics of indigenous languages where the emphasis is not so much on an ill-defined notion of competence but on the discovery, description and explanation of language practices and communicative repertoires that include speakers' perspectives in relation to their changing sociocultural realities.

Key words: language ideology, indigenous language competence, indigenous language speakers, monolingual bias, standard language.

\section{INTRODUCCIÓN}

Una tendencia que ha predominado en el estudio de la situación sociolingüística de los pueblos indígenas en Chile durante la última década ha consistido en tratar de determinar los niveles y tipos de competencia que los miembros de estos poseerían en sus lenguas. En el presente trabajo, mediante un examen articulado en torno al concepto de ideología lingüística, se plantea una lectura y una postura crítica respecto de esta tendencia con el objetivo de elucidar ciertos supuestos naturalizados y que han enmarcado la discusión académica en torno a dicha competencia, en particular, y a la situación sociolingüística de las lenguas indígenas, en general.

En el ámbito de los estudios de las lenguas indígenas en Chile, existen algunas investigaciones acerca del rol de las ideologías lingüísticas. Lagos, Espinoza y Rojas (2013) y Rojas, Lagos y Espinoza (2016) analizan las divergencias entre las ideologías lingüísticas de intelectuales (i.e., lingüistas) mapuche y las de los mapuche no especialistas y sus efectos, muchas veces contraproducentes, para procesos de planificación y revitalización de esta lengua. Lagos (2017) examina las ideologías lingüísticas presentes en comunidades pehuenches y su relación con los proyectos gubernamentales de Educación Intercultural Bilingüe en los colegios de la zona, mostrando que aquellas difieren de la tradición racional iluminista. Finalmente, Olate, Cisternas, Wittig y Flores (2017) analizan las ideologías lingüísticas en los trabajos de los frailes capuchinos alemanes, concluyendo que el discurso capuchino acerca de la lengua mapuche habría enfatizado la noción de lengua en peligro, promoviendo, además, estereotipos esencialistas de la relación entre lengua y cultura cuyos efectos perdurarían hasta hoy.

Hasta ahora, sin embargo, las ideologías lingüísticas presentes en los estudios elaborados por lingüistas acerca de la competencia en lengua indígena en Chile no habían sido exploradas. Esto se puede deber a que tales ideologías lingüísticas han sido asumidas como presupuestos científicos objetivos en la labor de especialistas que gozan de la legitimad y autoridad que les otorgan la institución y la práctica académica desde donde construyen sus discursos, así como la supuesta objetividad y transparencia de los enfoques cuantitativos que utilizan. 
Como se argumenta en este trabajo, si bien las investigaciones en torno a la competencia en lengua indígena se han realizado sin una definición adecuada de esta ni de los criterios empíricos para su medición, implícita y explícitamente despliegan una serie de ideologías lingüísticas que han moldeado una particular conceptualización y aproximación al fenómeno estudiado. Los supuestos que subyacen a estas ideologías lingüísticas actúan como soporte para describir y evaluar el uso lingüístico real de los sujetos estudiados y contribuyen a la naturalización de jerarquías de hablantes impuestas externamente.

El presente trabajo se organiza de la siguiente manera. En la sección 2 se desarrolla el concepto de ideologías lingüísticas, el cual informará la discusión en la sección 3. En esta, se identifican y caracterizan las ideologías lingüísticas presentes en los estudios de competencia y se discuten sus implicancias teóricas y metodológicas. En esta sección también se contrasta el discurso de estos estudios con la evidencia presente en otras investigaciones acerca de las situaciones de contacto lengua indígena-castellano en Chile. El trabajo concluye, en la sección 4, resumiendo los puntos principales del análisis, presentando otras reflexiones pertinentes que surgen de este y sugiriendo la necesidad de una aproximación diferente al estudio de la situación sociolingüística de las lenguas indígenas en Chile

\section{IDEOLOGÍAS LINGÜÍSTICAS}

Si bien las ideologías lingüísticas tienden a ser definidas de diversas maneras en la literatura especializada (Woolard 1998, Kroskrity 2004, del Valle y Meirinho-Guede 2016), para propósitos del presente trabajo se entenderán como conjuntos o sistemas de creencias y actitudes respecto de la naturaleza de las lenguas, los hablantes y los procesos comunicativos en general, así como los contextos en que estos ocurren, que permiten (o son utilizadas consciente o inconscientemente para) articular y sostener interpretaciones, evaluaciones y tomas de posición respecto de una serie de asuntos que, precisamente, atañen a las lenguas, sus usos y sus usuarios (Silverstein 1979, Rumsey 1990, Gal 1992, Woolard 1998, Schieffelin, Woolard y Kroskrity 1998).

Estas ideologías lingüísticas se producen y reproducen, de manera explícita o implícita, en una serie de prácticas lingüístico-comunicativas (textuales, discursivas) y metalingüísticas (Woolard 1998, del Valle 2007, Blommaert y Rampton 2011) y se enmarcan dentro de procesos sociales y culturales más amplios, ya que las "maneras de pensar sobre el lenguaje se articulan con ideologías sobre otros fenómenos" (Zavala 2016: 210).

Si bien las ideologías lingüísticas tienden a ser compartidas por los miembros de un grupo social o cultural determinado, esto no impide la existencia de heterogeneidad dentro de un mismo grupo (Kroskrity 2004). Sin embargo, el hecho de que estén asociadas a grupos concretos hace posible afirmar que existe una estrecha relación, en muchos casos, entre las ideologías lingüísticas y los intereses de estos grupos (Gal 1992, García, Flores y Spotti 2016): las ideologías lingüísticas serían el mecanismo mediante el cual los grupos sociales nombrarían y naturalizarían su particular manera de representar la realidad y el lenguaje 
(Gal 1992, Piller 2015) produciendo interpretaciones "como si de verdades inapelables se tratara" (del Valle y Meirinho-Guede 2016: 622). Esto abre las posibilidades del lenguaje para la manipulación social (Piller 2015), por ejemplo, a través de la producción de discursos especializados hegemónicos que naturalizan la manera de entender y estudiar una serie de fenómenos, en este caso, sociolingüísticos.

Entre los grupos cuya actividad también está moldeada por ideologías lingüísticas, en tanto aquella encarna los valores, creencias y actitudes de los espacios sociales e institucionales en los que "aprendieron a pensar y actuar" ${ }^{1}$ (Salö 2018: 25), y cuyas afirmaciones tienden a gozar de autoridad y legitimidad, están los propios especialistas en materias del lenguaje: lingüistas, antropólogos y sociolingüistas (Woolard 1998, Irvine y Gal 2000, Hill 2002, Kroskrity 2004, Ricento 2009, Paffey 2012, Rojas 2015, Fallas 2016 García et al. 2016, De Korne 2017, Lagos 2017, Kirsten 2017, Valdés 2017, Salö 2018). En este caso, las ideologías lingüísticas contribuirían a dar forma a una perspectiva profesional particular mediante supuestos acerca del lenguaje, la que influenciaría y se manifestaría en las prácticas teóricas, metodológicas y discursivas de estos especialistas, así como en sus evaluaciones de diversos fenómenos lingüísticos.

Como se mencionara, en este trabajo se analizan y discuten las ideologías lingüísticas presentes en el discurso hegemónico acerca de la competencia en lengua indígena en Chile. Siguiendo a Woolard (1998), el sitio apropiado para este estudio exploratorio se encuentra, en primer lugar, en el discurso metalingüístico presente en estas investigaciones. Este discurso se caracteriza por contener discusiones, evaluaciones y recomendaciones explícitas acerca de cómo los hablantes utilizan, o deberían utilizar, sus lenguas, y tiende a ser característico de los círculos expertos (Paffey 2012). Por otro lado, los supuestos teóricos y las decisiones y prácticas metodológicas presentes en estos estudios también arrojan luces acerca de las ideologías lingüísticas subyacentes en cada uno de ellos. Todo lo anterior está en línea con las propuestas de Blommaert y Rampton (2011) para quienes el examen exhaustivo del discurso puede revelar la manera en que las ideologías lingüísticas penetran el texto y el habla. En este análisis, siguiendo a Cifuentes (218:365), las formas lingüísticas son solo la entrada, ya que "el foco se hace en las ideas en sí mismas y la interpretación de estas en sus interrelaciones, es decir, cómo en conjunto conforman" una serie de ideologías lingüísticas en relación con el contexto mayor de los intereses y espacios discursivos de los grupos sociales involucrados.

En este trabajo se propone que un análisis de este tipo permite discutir cómo estas ideologías lingüísticas han moldeado los que, de otra manera, suelen ser entendidos como análisis objetivos en el campo de la sociolingüística de las lenguas indígenas en Chile, una idea que se ve reforzada por la preeminencia de los enfoques cuantitativos utilizados. En otras palabras, este análisis permite revisitar el trabajo académico existente y reexaminar una serie de conceptos y métodos recibidos, firmemente establecidos, y ya casi de sentido común y naturalizados (Blommaert 2006, Kirsten 2017). Otra ventaja de este análisis

${ }^{1}$ Todas las citas en inglés fueron traducidas por el autor. 
consiste en que, al situar el estudio de las ideologías lingüísticas en un grupo determinado con objetivos similares y cuyos miembros se mueven en un espacio discursivo (Heller 2009) compartido y creado en conjunto, podemos "identificar los intereses y espacios institucionales que posibilitan e incluso favorecen" la producción y les confieren sentido a las ideologías lingüísticas que sostienen y despliegan (del Valle y Meirinho-Guede 2016: 629). Constituye, así, un ejercicio de reflexividad epistémica, entendida como un ejercicio (auto)crítico de reflexión permanente para entender los supuestos que moldean nuestra generación de conocimiento en los espacios sociales e institucionales en que circulamos (Salö 2018).

\section{IDEOLOGÍAS LINGÜÍSTICAS EN EL ESTUDIO DE LA COMPETENCIA EN LENGUA INDÍGENA}

Una revisión al tratamiento teórico que ha recibido la noción de competencia en lengua indígena en los estudios que se han abocado a investigarla (Zúńiga 2007, Gundermann, González y Vergara 2007, Gundermann, Canihuán, Castillo y Clavería 2008, Gundermann, Vergara y 2009, Espinosa 2009, Lagos, Oyarzo, Mariano, Molina y Hasler 2009, Lagos 2012, Henríquez 2014, Kondic 2015, Alonqueo, Wittig y Huenchunao 2017, Zúñiga y Olate 2017) revela que estos no proveen ningún tipo de definición especializada ni modelo detallado y coherente de dicha competencia. El uso del concepto competencia es a tal punto confuso que dificulta comprender si se trata de competencia lingüística (por ejemplo de tipo chomskyana), competencia comunicativa (por ejemplo de tipo hymesiana), o de subcompetencias (de acuerdo, por ejemplo, a Canale y Swain 1980, Canale 1983a, Celce-Murcia 2007). Además, las definiciones de los niveles de competencia que a menudo se presentan (por ejemplo, competencia alta, intermedia y baja) no poseen mayor sustento teórico ni empírico. Finalmente, el uso confuso de un conjunto de términos y de distinciones, muchas veces ad hoc, revela la presencia de un metalenguaje bastante idiosincrático que no contribuye a entender claramente el fenómeno estudiado.

Esta falta de definición del fenómeno a estudiar, o su definición confusa, pone en entredicho la validez de los instrumentos utilizados en estos estudios. Esto, porque sin una adecuada definición de competencia es difícil determinar qué tipo de evidencia recolectar, cómo recolectarla, y cómo interpretar los resultados obtenidos (Canale 1988, Elder, McNamara, Kim, Pill y Sato 2017).

Un aspecto interesante que surge de la revisión de estos trabajos es que, a pesar de esta ausencia de un marco teórico coherente para el estudio de la competencia en lengua indígena, es posible identificar en ellos ideologías lingüísticas que han moldeado una comprensión compartida de este fenómeno. Estas ideologías lingüísticas generan ciertas expectativas por parte del investigador al momento de diseñar la investigación, ejecutarla e interpretar y evaluar los resultados obtenidos.

Dos son las ideologías lingüísticas identificadas en estos estudios: las ideologías monoglósica y de la lengua estándar. En las subsecciones siguientes se discute la manera 
en que estas ideologías se manifiestan y las implicancias teóricas y metodológicas que han tenido, y se contrastan las proposiciones presentes en estos estudios de competencia con la evidencia proporcionada por otras investigaciones de situaciones de contacto en Chile.

\subsection{Conceptualizaciones de la(s) lengua(s) y comunidad(es)}

Mientras que la ideología monoglósica se expresa mediante un sesgo monolingüe, la ideología de la lengua estándar se hace presente como una concepción de las lenguas en tanto sistemas reificados con una forma canónica uniforme (Milroy 2001, Blommaert 2006, García 2009, García y Vergara 2010, Allard et al. 2014, Flores y Rosa 2015, McKinney et al. 2015, Wardhaugh y Fuller 2015, Poza 2016, Rosa y Burdick 2016, Ganuza y Hedman 2017). Así, en estos estudios las lenguas son entendidas como sistemas cerrados estables y estáticos, jamás afectadas por procesos de innovación o contacto, con una norma monolingüe estándar claramente identificable (y fácilmente asociable a comunidades específicas) donde la diversidad y variabilidad interna no tendrían lugar, y cuya existencia en esta forma pura e idealizada es independiente de sus contextos de uso y usuarios.

De esta manera, es posible plantearse objetivos tales como describir la situación de una lengua (a saber, la lengua indígena) (por ejemplo, Zúniga 2007, Gundermann et al. 2008, Espinosa 2009, Gundermann et al. 2009, Lagos et al. 2009, Lagos 2012, Henríquez 2014, Kondic 2015, Alonqueo et al. 2017). Estos objetivos así planteados imponen a priori una separación entre las lenguas y un sesgo monolingüe: ocultan la posible fluidez de las prácticas comunicativas bilingües a nivel individual y social e imponen la idea de que la comunicación prototípica es por defecto monolingüe. Igualmente, estos estudios necesariamente presuponen la existencia de lenguas indígenas estandarizadas en Chile, y se orientan al modelo estándar del castellano (ver Olate 2017a al respecto).

Los instrumentos utilizados también reflejan esta visión de las lenguas e imponen a los entrevistados una manera de interpretar su propia situación sociolingüística. Estos instrumentos están diseñados para medir competencia en una lengua o en la otra, más que para capturar las dinámicas comunicativas reales de los hablantes (bilingües de diferente tipo en muchos casos). Esto se demuestra cuando se pide a los entrevistados decir si hablan o no hablan una lengua, o cuál lengua hablan en determinados dominios y con quién. $\mathrm{O}$ cuando se les pide a individuos bilingües que separen sus lenguas para funcionar en el modo monolingüe que exigen los investigadores en una entrevista. Finalmente, también se observa en la interpretación de los resultados cuando, por ejemplo, el desplazamiento lingüístico se ve como un proceso simplificado de reemplazo de una lengua por otra.

Estas ideologías, así, permiten desprender a las lenguas de los hablantes y de sus realidades comunicativas (Davis 2017, De Korne 2017). Davis (2017: 39) se refiere a este proceso como extracción lingüistica, la cual consistiría en discutir las lenguas extrayéndolas de las vidas personales, las prácticas comunicativas, las comunidades y las experiencias en las cuales están insertas. Por esto, los estudios revisados también tienden a suponer una comunidad de habla ahistórica, homogénea y perfectamente diglósica (o monolingüe) 
Estos estudios ignoran que muchas veces ciertos contextos no requieren de los hablantes los conocimientos por los que los investigadores preguntan. Es posible afirmar que, de conocer tales realidades, ninguno de los entrevistados sería comunicativamente incompetente, ya que la pertenencia a una comunidad se sustenta sobre la base de normas interaccionales compartidas, las cuales no son siempre monolingües ni implican la posesión de todas las lenguas presentes en una comunidad (Hymes 1992, Romaine 2000). ${ }^{2}$ Así, lo correcto o apropiado lingüística y comunicativamente no se puede determinar sin hacer referencia al repertorio de la comunidad de la que es parte un individuo. Olate (2012) desarrolla esta idea bajo la noción de competencia lingüístico-comunicativa situada, que considera las dinámicas sociolingüísticas de una comunidad para la comprensión de un fenómeno lingüístico determinado. Contextualiza e historiza, así, las formas y las funciones lingüísticas, las que surgen desde la realidad comunicativa de los propios hablantes más que de un modelo de lengua, comunicación y comunidad idealizado en la mente del investigador.

\subsection{Conceptualización de hablantes, competencia y bilingüismo}

La ideología monoglósica favorece al monolingüismo como la norma y el ideal comunicativo por defecto. Los hablantes ideales son vistos como monolingües (aunque sean bilingües) con una competencia completa y perfecta en sus lenguas, a la cual todos los sujetos del grupo étnico debiesen aspirar para ser clasificados como hablantes propiamente tales (García y Vergara 2010, Allard et al. 2014, Flores y Rosa 2015, Poza 2016, McKinney 2016, Vogel y García 2017, Rosa y Burdick 2016). Este es otro aspecto problemático para el caso chileno, ya que algunos de los estudios revisados afirman que los hablantes monolingües de lenguas indígenas son prácticamente inexistentes (Gundermann et al. 2007, Gundermann et al. 2008, Espinosa 2009, Kondic 2015). Esto, sin embargo, parece no afectarles, ya que, así como descontextualizan a los hablantes, las ideologías lingüísticas presentes en estos estudios les permiten operar independientemente de que la norma monolingüe utilizada para evaluar, así como sus representantes, no pueda ser localizada empíricamente (Flores y Rosa 2015).

Al ser los hablantes monolingües con una competencia completa y perfecta en su lengua la norma, es posible construir los cuestionarios de autoevaluación utilizando un metalenguaje que recurre a conceptos como competencia alta, mediana y baja (lo que refuerza la idea de hablantes completos e incompletos). Esto también permite utilizar

\footnotetext{
2 Para Hymes (1992: 46) "la membresía en una comunidad nunca es reducible a características únicamente lingüísticas”. El ejemplo que cita Romaine (2000: 24) es el de comunidades bilingües escocés/inglés donde hay hablantes que solo tienen una competencia receptiva en escocés pero que sin embargo son capaces de participar en interacciones con hablantes de dicha lengua. Para esta autora, esto es una demostración de que poseen competencia comunicativa. No es difícil imaginar que en una serie de comunidades indígenas en Chile también se puede dar esta situación. Por ejemplo, los participantes en el estudio de Olate, Wittig y Hasler (2014) son "escolares mapuches monolingües de espańol que habitan una comunidad bilingüe".
} 
expresiones como hablar mejor o peor o entender todo, mucho, bastante, o poco (lo que refuerza la idea de hablantes perfectos e imperfectos). Esto también queda de manifiesto en aquellos estudios que utilizan las llamadas mediciones directas, donde la norma monolingüe absoluta e ideal la representan los entrevistadores (Gundermann et al. 2008, Espinosa 2009) o la poseen los investigadores (Lagos et al. 2009, Lagos 2012, Alonqueo et al. 2017).

Esta ideología monoglósica también conceptualiza al bilingüismo como monolingüismo paralelo y excluyente, y al individuo bilingüe como dos monolingües en uno (lo que refuerza el mito del bilingüismo balanceado). Esta perspectiva hace posible pedirle a un individuo bilingüe que separe y controle sus conocimientos y habilidades en cada lengua, para así ser evaluado de acuerdo a la norma monolingüe imaginada (García 2009, Allard et al. 2014, McKinney et al. 2015, Poza 2016, Ganuza y Hedman 2017, Vogel y García 2017). Tal perspectiva es posible identificarla en las mediciones indirectas utilizadas (por ejemplo, Zúñiga 2007, Gundermann et al. 2007, Gundermann et al. 2008, Espinosa 2009, Lagos 2012), donde las lenguas son entendidas como entidades mutuamente excluyentes las que los sujetos saben o no. Las mediciones directas (Gundermann et al. 2008, Espinosa 2009, Lagos et al. 2009, Alonqueo et al. 2017) también favorecen esta visión del bilingüismo, ya que buscan forzar un comportamiento comunicativo monolingüe por parte de individuos bilingües con el fin de determinar su nivel real de competencia en una lengua. La validación de esta posibilidad se realiza mediante el trabajo del entrevistador: sujeto bilingüe capaz de conducir una entrevista en modo monolingüe. Así, parece lógico y legítimo pedir lo mismo a los entrevistados.

Las interpretaciones que luego se hacen refuerzan estas ideas, por un lado, entregando cifras de hablantes de lengua indígena (desconociendo su carácter de bilingües), o categorizándolos en niveles de competencia, o reforzando la visión monoglósica del bilingüismo. Por ejemplo, Espinosa (2009: 45) al contar a los hablantes de aymara nos dice que serían 115 . De estos, tres habrían declarado hablar solo aymara y 112 habrían declarado ser bilingües. Así, literalmente divide a estos hablantes bilingües en dos monolingües para contar al monolingüe aymara en ellos $(112+3=115)$. Gundermann et al. (2008: 100) describen a los sujetos que categorizan como bilingües en su estudio como "hablantes que separan ambos idiomas a la hora de estructurar un discurso. Hay un mayor control entre las dos lenguas". ${ }^{3}$

\footnotetext{
${ }^{3}$ Tal ideología también se encuentra presente en el discurso oficial, a través de los textos entregados por MINEDUC para la enseñanza de lenguas indígenas, donde se plantea que "Los y las estudiantes, al ser bilingües, acceden a dos sistemas de códigos o formas de representación de la realidad que no se confunden entre sî" y que "la confusión entre dos códigos puede afectar de distintas formas la comprensión y expresión lingüística en una de las dos lenguas" (MINEDUC 2017: 8-9). También se encuentra presente en discusiones en torno a la planificación lingüística de las lenguas indígenas. Por ejemplo, Chiodi y Loncon (1999), al discutir instancias en que los niños mezclan ambas lenguas, atribuyen tal comportamiento a una incapacidad de los niños para reconocer los límites de cada código lingüístico, lo que, nos explican, también sería un problema de los padres, quienes no separarían dichas lenguas al momento de hablarles. Esto último, aunque estos autores no lo mencionen, es solo una inferencia, puesto que en su trabajo en ningún momento analizan interacciones padres-hijos.
} 


\subsection{Evaluación de las prácticas comunicativas y conocimiento reales}

La consecuencia de tomar como modelos a la lengua estándar y a una especie de hablante monolingüe pre-contacto lingüístico, es que todo lo que no se aproxime a tales modelos es evaluado como comportamiento deficitario o como problema (Moore, Pietikäinen y Blommaert 2010, De Korne 2017, Fallas 2016). Tal es el caso de los estudios de competencia revisados.

Gundermann et al. (2008: 100) nos dicen de un tipo de hablantes que "si bien tiene un alto manejo del mapuzugun, presenta a la vez un español altamente interferido cuando estructuran frases en este idioma, ... [serían] bilingües subordinados [ya que] en la segunda lengua tienen un control menor que en la lengua materna" (cursivas mías).

Los autores no solo entienden a los participantes como hablantes imperfectos sino que, además, desde su perspectiva monoglósica, sostienen la idea de una especie de bilingüismo balanceado como el ideal ${ }^{4}$, una noción que los estudios en bilingüismo han desechado como un mito (Baker y Jones 1998, Dewaele 2011). Estos mismos autores, luego nos dicen de los bilingües pasivos que estos "no logran estructurar respuestas" (p. 101). El ejemplo que dan es el siguiente:

\section{Extracto $1 . .^{5}$}

Encuestador: Mari mari, inche Daniel pigen, ¿eymi iney pigeymi? Entrevistado: Rosa

Encuestador: mapuchezugukeymi?

Entrevistado: más o menos no más

Encuestador: fey tamu müley miawi lamgen?

Entrevistado: may

Encuestador: chumgechi tami mapu, tami ruka?

Entrevistado: kümelkaley

Encuestador: chem mew ta küzawküleymi lamgen?

Entrevistado: küzawkelhan, no

Encuestador: küzawkelhaymi?

Entrevistado: no, dueña de casa no más

(Gundermann et al. 2008: 101)

\footnotetext{
${ }^{4}$ Sin mencionar que se presume la transparencia de expresiones del tipo "alto manejo" de un idioma y se entiende al uso del lenguaje para propósitos comunicativos como "estructurar frases".

${ }^{5}$ En este extracto y en los siguientes, los textos son citados según aparecen en los estudios originales. Es necesario aclarar que, más allá del conocimiento de la lengua mapuche que en ciertos casos desplieguen los entrevistadores en algunos de estos estudios, mi argumento se centra en las implicancias de aproximarse al estudio de la competencia en lengua indígena de los entrevistados desde una perspectiva que impone el monolingüismo.
} 
Según los autores, "En este caso la entrevistada, de edad mediana... aunque entiende muy bien el mapuzugun, no responde en este idioma sino en castellano" (p. 101) (cursivas mías). ¿Por qué "aunque"? ¿Quién determina que la entrevistada tendría que responder en mapudungun? ¿Por qué no basta con que entienda muy bien la lengua indígena? Así, este uso real es un problema comunicativo solo desde la visión de los investigadores, quienes exigen un comportamiento monolingüe absoluto en una situación artificial a individuos que en sus vidas reales parecieran no comportarse como tales. Las evaluaciones negativas también se hacen en el caso de los sujetos a quienes estos autores denominan bilingües incipientes, ya que estos tendrían un conocimiento elemental y no automático, cuyas respuestas, nos dicen, suelen ser incorrectas.

Otro extracto interesante es el siguiente:

Extracto 2.
Encuestador: ¿eymi kimimi ta chem pigey pi ta awelu paterno?
Entrevistado: ¿awelu paterno?
Encuestador: may, ¡chem pigey?
Entrevistado: chem pigey pi ta... mmm... awelu!
Encuestador: chem pigey pi ta tío materno, ¿chem pigekey chezugunmu?
Entrevistado: ahí no estoy...

(Gundermann et al. 2008: 111)

De este entrevistado nos dicen que sería un hablante con alta "competencia lingüística en mapuzugun, sin embargo, en su repertorio lingüistico no está la distinción que el idioma tiene para conceptualizar el parentesco y acude al uso del genérico abuelo propio del idioma castellano" (p. 112) (cursivas mías). Acá, no basta con la "alta competencia lingüística" ni con la presencia de fenómenos extremadamente comunes en situaciones de contacto lingüístico prolongado. Lo que realmente importa a los investigadores son las distinciones "que el idioma tiene"

Para Lagos et al. (2009: 124) los participantes son descritos como incapaces o incompetentes al no poder realizar "algún componente básico" de la tarea que les presentaban enteramente en mapudungun. Pero no explican si tal tarea y la lengua en que debían realizarla reflejaban alguna situación comunicativa real y necesaria para los participantes, criterio necesario para referirse a su competencia comunicativa.

\footnotetext{
${ }^{6}$ Por lo demás, nótese que utiliza la expresión awelu, y no abuelo, como dicen los investigadores. Awelu paterno, además, ya había sido utilizada por el propio entrevistador (quien también utiliza tío materno).
} 
Otro ejemplo lo encontramos en Espinosa (2009), donde se transcribe la siguiente producción de una de las entrevistadas:

Extracto 3.

Naya ganaderot sapuru alpachu awatista, uka qulqimpi wawanaka estudiyaskt, eso... Naya gustitaspawa, mejoraña, alpachuña, ukat parwaxa llawirasiña ch'ankanaka aljasiña, chukutanaka aljasińa, ukampi wawanaka estudiayaña... (risa). No lo entiende eso... En aymara ¿no?, mi esposo sacrificio lurańa thayampi, jallumpi ... wali ch’ama, samaqaña, manq'ata jan manq'ata uka uywampi.

(Espinosa 2009: 51)

Nuevamente, a pesar de que en este extracto se aprecia claramente el resultado del contacto lingüístico, para la autora esto demostraría que el aymara hablado en Chile es una variedad no fluida y con presencia de formas híbridas. En definitiva, un sistema altamente erosionado, a diferencia de un aymara puro. Sin embargo, esta es una producción real de un hablante real que constituye una manifestación clara de la manera en que los hablantes utilizan los recursos comunicativos de los que disponen.

Estos comentarios por parte de los investigadores reducen estos extractos a evidencia de que los sujetos hablan mal (ya que no se comportan como el monolingüe ideal) y no a evidencia de usos lingüísticos legítimos originados en situaciones prolongadas de contacto asimétrico y que aún están por descubrirse, describirse y explicarse.

Alonqueo et al. (2017), en tanto, si bien entregan ejemplos de la producción de los niños participantes en su estudio con el fin de ilustrar sus niveles de competencia en mapudungun, al categorizarlos en estos niveles (que reflejan e imponen una norma monolingüe) en efecto terminan marginalizando sus reales comportamientos comunicativos como estos se manifiestan en su vida cotidiana (que es donde se despliega la competencia comunicativa). Claramente, dados los mismos ejemplos utilizados por los investigadores, es posible inferir que algunos niños usan y entienden más o menos mapudungun. Así, el rendimiento en la prueba utilizada por estos autores sirve para validar categorías y crear jerarquías externas que pueden no ser significativas para la comunidad, y no para revelar usos reales del lenguaje que nos pueden entregar pistas importantes para comenzar a describir y comprender las prácticas comunicativas (más que la competencia individual) que han ido surgiendo a través de los diferentes tipos de interacción en estas comunidades.

El conocimiento de los hablantes también es interpretado como manifestación de déficit. En una de las tareas en Espinosa (2009) a los entrevistados se les pidió escuchar una grabación en aymara. La autora explica que los entrevistados asignaron la pronunciación en la grabación a un aymara de Bolivia. Para ella, "Esta impresión es común entre hablantes de 
aymara chilenos, cuando escuchan una variedad de lengua bien estructurada y pronunciada" (p. 50). En otras palabras, cuando escuchan hablar bien. Con esto, no solo refuerza las visiones de lengua discutidas hasta ahora, sino que desconoce que tales comentarios de sus entrevistados revelan un conocimiento profundo, que se puede traducir en algo así como él no habla como yo lo hago y, por lo tanto, no es de mi comunidad (y también reflejan como en los hablantes es posible encontrar ideologías puristas). Tal conocimiento surge de la experiencia de los sujetos y demuestra que son capaces de percibir diferencias y crear categorías y límites reales y con sentido para ellos.

La construcción de los entrevistados como sujetos no conocedores también queda de manifiesto en los argumentos para justificar las mediciones directas, que destacan el hecho de que los entrevistados pueden ser imprecisos al momento de declarar su propia competencia o la de terceros. Este argumento es válido, ya que esta es una de las dificultades que presentan las entrevistas de autodeclaración de conocimiento (Pauwels 2016, Alonqueo et al. 2017, pero ver Fishman 1991: 52). El problema surge cuando el investigador, en vez de diseñar con mayor cuidado los cuestionarios y limitar sus interpretaciones a los datos así recogidos, diseña mediciones directas como un mecanismo de control para determinar con mayor precisión (supuestamente) aquello que los entrevistados no serían capaces de expresar correctamente.

Estos estudios, así, no solo borran aquello que complejizaría la realidad estudiada (Irvine y Gal 2000), sino que, además, interpretan el comportamiento de los hablantes como incorrección, falta de competencia, o comportamiento deficitario (McKinney et al. 2015) condenándolos a "habitar un lugar definido por la incompletitud, la inadecuación y el déficit” (Fallas 2016: 250).

\subsection{El discurso de la competencia en lengua indigena y el contacto lingüistico}

Las ideologías lingüísticas presentes en los estudios en torno a la competencia en lengua indígena han contribuido a construir discursos que ignoran las realidades sociolingüísticas y lingüísticas presentes en las situaciones de contacto asimétrico en Chile, las que apuntarían, en algunos casos, a la existencia de variedades que se alejarían de una supuesta norma estándar y, en otros, a situaciones de heteroglosia en el uso lingüístico. Estas situaciones son características en contextos donde los hablantes recurren a un repertorio diverso de recursos comunicativos (incluyendo diversas lenguas) en sus interacciones cotidianas (Kyratzis, Reynolds y Evaldsson 2010). Tal afirmación se puede sustentar en una serie de otros estudios ${ }^{7}$ (además de los ejemplos de la sección anterior).

En primer lugar, están aquellos estudios que han investigado el contacto a nivel estructural. Hernández y Ramos (1978) afirman que el castellano hablado por los niños

\footnotetext{
${ }^{7}$ En esta subsección no se discuten las perspectivas teóricas desde las cuales se realizaron estos estudios (lo que, de hecho, podría revelar muchas de las cuestiones ya discutidas). Más bien, se utiliza la evidencia recogida por estos estudios como una muestra clara de la existencia de prácticas comunicativas híbridas y fluidas, en ningún caso enteramente monolingües en una lengua estándar. Los ejemplos de cada artículo son solo una muestra de lo que cada autor expone en cada caso.
} 
mapuche en su estudio mostraría fuertes transferencias del mapudungun a nivel fonológico, gramatical y léxico, por lo que se apartaría de las normas del castellano estándar de Chile. Álvarez-Santullano y Contreras (1995: 184, en Olate 2012: 138) afirman que "el castellano hablado por estos informantes huilliches presentará rasgos particulares, derivados del contacto histórico con el tsesungun". Espinosa (2003) estudia lo que denomina español andino en niños aymara. Afirma, entre otras cosas, que la morfosintaxis del español andino chileno mostraría rasgos atribuibles a la actuación del aymara. Para el caso del castellano en contacto con el mapudungun, Olate (2012: 140) presenta un cuadro resumen para el nivel morfosintáctico de este castellano, de acuerdo a lo descrito por numerosas investigaciones (ver también Olate 2017a para una revisión detallada de estos estudios). Finalmente, Olate, Wittig y Hasler (2014: 185) proponen en su estudio que "en el español hablado por niños mapuches monolingües conviven rasgos derivados de dicho monolingüismo y de la escolarización ...con rasgos propios del castellano mapuchizado hablado por bilingües... con predominio de mapudungun". 8

En segundo lugar, respecto del estudio de las prácticas comunicativas desde una perspectiva sociolingüística, los estudios aún son escasos, pero los pocos existentes apuntan a prácticas heteroglósicas y fluidas. Por ejemplo, Sepúlveda (1984: 228) afirmaba, entre otras cosas, que sus entrevistados mapuche "reconocen "hablar una mezcla", es decir, pasan de un idioma a otro sin restricciones". En el estudio de Durán y Ramos (1989) también es posible inferir que en los hogares de las alumnas estudiadas se daban diferentes tipos de intercambios bilingües, y que en ningún caso constituían espacios completamente diglósicos.

Además, si se revisan las trascripciones de grabaciones del habla de los participantes en algunos estudios, es posible observar la existencia de variados tipos de comportamiento híbrido, jamás una norma idealizada monolingüe. Hermosilla (1998) muestra las interacciones comunicativamente exitosas entre una madre y su hijo:

Extracto 4 .

1) FR: Pekumunenge pichike patu

MA: no, no están

(Hermosilla 1998: 172)

2) FR: ¿Tiene sueño Manuel? Küpaumawimi

MA: Kudupuayu müten

FR: Vamos acostarnos

MA: Dewi

(Hermosilla 1998: 174)

${ }^{8}$ En mi búsqueda bibliográfica no logré encontrar estudios cuyo foco sean los cambios en las lenguas indígenas en situación de contacto en Chile. 
Chiodi y Loncon (1999) presentan el siguiente extracto de una entrevista, donde los niños evidencian la hibridez de sus prácticas comunicativas cotidianas (lo que dichos autores ven como un problema):

Extracto 5.

a.-Ya Juanito, ¿cem niey ta ni huerta? ¿Cumley tami huerta, cem muey, pvjeley?

b.-May pvjeley.

a.-Cew.

b.-Eh, a la orilla del pozo.

a.-Ya, ka cem ta mvley.

b.-Kopo, ka trapi, tomate, eh, eh, tene rabanito igual, napor, ka cem ta nie te lechiga ka cebolla.

(Chiodi y Loncon 1999: 20)

Makihara (2005), en su estudio acerca de las formas Rapa Nui de hablar español, nos presenta el siguiente ejemplo de una conversación entre padre e hija, que revela el uso fluido del repertorio lingüístico de estos hablantes:

Extracto 6.

Father: Ko tu'u mai 'ana ho'i tū pu'ahu era mai $i$ a Tire. Te zapatia, te $=$

Daughter: $=$ Pero papi, yo no recibí ni un este $=$

$\overline{\mathrm{F}}:=$ 'Ina ia a mamita kai hana mo hakatake'a atu. Ahiahi nō ka hakatake'a iho atu.

D: Pucha! ¿Porqué no me mostró? No me dijo.

$\overline{\mathrm{F}:}$ Ki tu'u nō koe mai te hāpī.

D: Papi! Pero si yo le pregunté por el paquete y me dijo que todavia no llegaba.

F: Ki tu'u nō koe i te ahiahi e mo ai o Tavake takoa, ararua kōrua 'ana mataki $i$ te pu'ahu era, ta'e hoko tahi nō koe.

D: iEn la tarde lo abrimo?

F: Ki tu'u a Tavake mai te hāpī. 
En Relmuan (2005) aparecen los siguientes extractos de entrevistas, donde ambas lenguas se utilizan fluidamente:

\section{Extracto 7.}

[Extracto de entrevista a comunero hablando de las comunidades]

"Fuera la comunidad fey ta doy, ka doy füta Organización ta trawüluwi che, trawüluwi fill lof [...] Aquí por José Llancao pingey por Llamuco, todo eso fey ta lof Pircumche pingey, pütrüley lof ta mu. Parece que fey ta püle doy pütrüley lof, más que en ninguna parte parece pu.[...] Müley Treupan pingelu, empézalu por Truf Truf Chureo-Sandoval pingey, diferente lof müley".

(Relmuan 2005: 81)

[Extractos de entrevista a comunero explicando el uso de las lenguas]

"Lof che mapudungun ka wingkadungulelkeiń. Mapuchedungukeiń may, chedunguyiń müten ruka mu, faleiń. Fey ta toca uno se va en el pueblo uh..ahí deja de hablar. Lof che mapudungun ka wingkadungulelkeiñ. Mapuchedungukeiñ may".

"Mapuche ka ta ti weke che fey ta doy wingkadunguy, pero ya ńochika kimnierpuy ka. Porque kińeke familia ta, ta chi lof re wingka dungun ta enseńaluwi”.

(Relmuan 2005: 89)

Finalmente, en Ojeda y Álvarez (2014), los entrevistados también desplegaron un comportamiento que se aleja de la norma monolingüe ideal:

\section{Extracto 8 .}

"Mucha gente nueva wekeche cambia letuykimtulaydew chedungun ..., entonces así estamos perdiendo como, como le dijera porcionada de mapuche" (E2).

(Ojeda y Álvarez 2014: 174)

Esta no pretende ser una lista definitiva de contextos sociolingüísticos y de las prácticas comunicativas presentes en ellos (por ejemplo, en Pozo 2014 y en Teillier $e t$ al. 2016 aparecen extractos de entrevistas que se orientan hacia un extremo más monolingüe en mapudungun). El objetivo más bien es destacar que esta evidencia es ignorada por los estudios revisados, los cuales simplemente construyen (imaginan) una norma de comportamiento 
monolingüe ideal en una comunidad diglósica ideal, desconociendo fenómenos comunes en situaciones de contacto. Algo similar plantean Wittig y Olate (2016: 129, ver también Olate 2017b) en su discusión acerca de la oficialización del mapudungun, cuando indican que "los estudios sociolingüísticos dan cuenta del estado de la lengua y olvidan el proceso de interacción que ha vivido" y que muchos activistas "de la lengua también suelen enfocarse en el estado, desconociendo la dinámica de cambio a la que está sujeta en manos de los hablantes".

\section{ReFLEXIONES FinALES}

A pesar de la ausencia de una teoría y metodología coherentes en los estudios en torno a la competencia en lengua indígena, todos los trabajos revisados despliegan ideologías lingüísticas comunes. Estas ideologías han moldeado una particular conceptualización de dicha competencia y de una serie de fenómenos relacionados, así como la manera de estudiarlos y evaluarlos. También han contribuido a naturalizar la categorización de los sujetos como competentes o incompetentes, al imponer externamente una norma idealizada monolingüe y una visión de las lenguas como sistemas autónomos y estandarizados, lo que, además, se ha hecho ignorando las comunidades de las que los hablantes son parte así como el real uso de su repertorio lingüístico, el cual incluso ha sido recogido en algunos de estos mismos estudios.

El hecho de que en todos los estudios revisados las ideologías lingüísticas sean similares, requiere de un análisis específico para definir cómo se han ido configurando y socializando estas concepciones acerca del lenguaje en Chile en los círculos especializados, específicamente en torno a los estudios en lenguas indígenas. También sería interesante determinar hasta qué punto la existencia de estas ideologías lingüísticas se ha reforzado debido a la falta de innovación metodológica, expresada en la sobreutilización de encuestas y entrevistas y de enfoques cuantitativos, los que han alejado a los investigadores de los contextos reales de comunicación de los hablantes, los cuales tienden a ser asumidos más que empíricamente investigados.

La evidencia de otros estudios en torno a las situaciones de contacto, ha permitido contrastar las proposiciones contenidas en los estudios de competencia con lo que parece ser una realidad claramente heteroglósica, caracterizada por un comportamiento lingüístico fluido e híbrido, común en situaciones bilingües y el cual no implica necesariamente problemas comunicativos. Estas realidades aún están por documentarse e investigarse en Chile.

Como efecto de las ideologías lingüísticas que lo han moldeado, el estudio de la competencia en lengua indígena, más que contribuir a la comprensión de los repertorios de los hablantes y de sus variadas y dinámicas realidades comunicativas, solamente ha servido para promover ciertas conceptualizaciones particulares del lenguaje (las de los investigadores), generar jerarquías de hablantes (impuestas externamente), marginalizar prácticas comunicativas reales y reducir a números realidades sociolingüísticas complejas y 
diversas. Respecto de esto último, la preponderancia de números es funcional a las ideologías discutidas en este trabajo, ya que, para contar a las lenguas y a sus hablantes, por ejemplo, es necesario entenderlos como entidades estáticas y autónomas (Moore et al. 2010).

La tendencia impuesta por estos estudios debe ser superada. Las posibilidades y retos que se presentan son muchos y complejos, y acá solo se pueden esbozar brevemente. En primer lugar, implican reformulaciones teóricas en torno a los fenómenos a estudiar y a su pertinencia, que vayan más allá de la actual obsesión con una vagamente definida competencia en lengua indígena. En este sentido, e independientemente de los temas que pueden ser objeto de estudio, es necesario superar las visiones modernistas y estructuralistas del lenguaje y la sociedad, las que claramente no permiten dar cuenta de las complejidades presentes en las situaciones de contacto y desplazamiento en la actualidad en Chile. El reto también consiste en reflexionar acerca de las maneras en que conceptualizamos el lenguaje, desde donde lo conceptualizamos, en relación con qué tipo de evidencia, y a quién sirven dichas conceptualizaciones, con el fin de generar modelos más inclusivos (Teillier 2013, García et al. 2016) y dinámicos, cuyo poder descriptivo y explicativo también tenga sentido para las comunidades estudiadas (por ejemplo, para contribuir efectivamente a los procesos de planificación y revitalización). Estos avances teóricos no se pueden realizar como hasta ahora en función de comunidades imaginadas (Zemelman 2001), sino que deben alinearse con el estudio de "las prácticas lingüísticas en relación con las condiciones socio-históricas, políticas y económicas que las producen" (García et al. 2016: 5).

Metodológicamente, parece ser momento de superar, o al menos moderar y mejorar, el uso de cuestionarios y enfoques cuantitativos. A manera de sugerencia inicial y para nada exhaustiva, pueden contribuir en este sentido la incorporación de estudios de caso con enfoques cualitativos o mixtos de comunidades, familias o individuos; la realización de trabajo de campo prolongado, con observación (participante o no), que permita entregar descripciones más complejas y detalladas de las comunidades estudiadas y sus usos lingüísticos, las que tengan algún grado de realidad para los miembros de estas y que permitan hacer comparaciones a través de casos (ver, por ejemplo, el proyecto de documentación sociolingüística descrito en Childs, Good y Mitchell 2014, también las propuestas de Olate 2017b); la grabación en audio y video de prácticas comunicativas reales, que nos permitan dar cuenta de manera detallada de los cambios que van ocurriendo en situaciones de contacto y desplazamiento. Este tipo de evidencia permitiría evitar que nuestras discusiones se realicen en "oposición a cualquier característica objetiva de[1] uso lingüístico” (Flores y Rosa 2015: 151), como ha ocurrido hasta ahora.

Lo anterior también implica la adopción de una visión crítica que no se conforme con la descripción (por muy detallada que esta sea), sino que indague en la manera en que diversas relaciones de poder han contribuido a la configuración de estas situaciones sociolingüísticas y las consecuencias reales que esto pueda tener para la vida de los hablantes. Finalmente, es necesaria la elaboración de protocolos éticos para trabajar con y en las comunidades cuyo desarrollo es imposible si no nos acercamos e involucramos activamente con ellas. 
En conclusión, es necesario promover una sociolingüística de las lenguas indígenas cuyo énfasis no sea la producción de categorías y porcentajes de hablantes competentes e incompetentes, lo que solo tiene realidad en cierto discurso académico especializado y que contribuye a la (re)marginalización de grupos minorizados. Los objetivos deben ser más bien el descubrimiento, la descripción y comprensión de las prácticas lingüísticas y los repertorios verbales y comunicativos (Gumperz 1964 Busch 2012) de -y desde- los hablantes (Teillier 2013), en relación directa con sus complejas y cambiantes realidades sociales, culturales y comunicativas, incorporando dimensiones políticas e históricas para comprender de mejor manera las fuerzas y sujetos que van moldeando estas realidades y las consecuencias (no solo) lingüísticas y sociolingüísticas de esto.

\section{OBRas Citadas}

Allard, Elaine, Katherine Mortimer, Sarah Gallo, Holly Link y Stanton Wortham. 2014. "Immigrant Spanish as liability or asset? Generational Diversity in Language Ideologies at School". Journal of Language, Identity \& Education 13,5: 335-353.

Alonqueo, Paula, Fernando Wittig y Nataly Huenchunao. 2017. "Lleupeko tuwün. Un estudio exploratorio sobre niveles de competencia en mapuzungun en niños mapuches de la Araucanía". Alpha (Osorno) 44: 119-135.

Baker, Colin, y Sylvia Prys Jones, eds. 1998. Encyclopedia of Bilingualism and Bilingual Education. Clevedon, Inglaterra: Multilingual Matters.

Blommaert, Jan. 2006. "Language ideology". Encyclopedia of Language and Linguistics, Vol. 6. Ed. Keith Brown. Oxford: Elsevier. 510-522

Blommaert, Jan y Ben Rampton. 2011. "Language and Superdiversity”. Diversities 13, 2: $1-21$

Busch, Brigitta. 2012. “The Linguistic Repertoire Revisited”. Applied Linguistics 33, 5: 503523.

Canale, Michael. 1983a. From Communicative Competence to Communicative Language Pedagogy. Language And Communication. Eds. Jack C. Richards y Richard W. Schmidt. New York: Longman. 2-27.

Canale, Michael. 1988. "The Measurement of Communicative Competence". Annual Review of Applied Linguistics 8: 67-84.

Canale, Michael, y Merrill Swain. 1980. "Theoretical Bases of Communicative Approaches to Second Language Teaching and Testing." Applied linguistics 1, 1: 1-47.

Celce-Murcia, M. 2007. Rethinking the Role of Communicative Competence in Language Teaching. Intercultural Language Use and Language Learning. Eds. Eva Alcón Soler y María Pilar Safont Jordá. Berlin: Springer. 41-57.

Childs, Tucker, Jeff Good y Alice Mitchell. 2014. "Beyond the Ancestral Code: Towards a Model for Sociolinguistic Language Documentation”. Language Documentation and Conservation 8:168-191. 
Chiodi, Francesco y Elisa Loncon. 1999. Crear nuevas palabras. Innovación y expansión de los recursos lexicales del mapuzugun. Temuco: UFRO - CONADI.

Cifuentes, Juan. 2018. "Ideologías lingüísticas en Chile: El Boletín de la Academia Chilena de la lengua." Logos 28, 2: 361-376.

Davis, Jenny. 2017. "Resisting Rhetorics of Language Endangerment: Reclamation Through Indigenous Language Survivance". Language Documentation and Description, vol 14. Ed. Wesley Y. Leonard y Haley De Korne. Londres: EL Publishing. 37-58

De Korne, Haley. 2017. "The multilingual realities of language reclamation: Working with Language Contact, Diversity, and Change in Endangered Language Education”. Language Documentation and Description. vol 14. Ed. Wesley Y. Leonard y Haley De Korne . Londres: EL Publishing. 111-135

Del Valle, José. ed. 2007. La lengua, ¿patria común? Ideas e ideologías del español. Madrid/ Frankfurt: Iberoamericana/Vervuert.

Del Valle, José, y Vítor Meirinho. 2016. Ideologías lingüísticas. Enciclopedia de lingüística hispánica. Vol. 2. Ed. Javier Gutiérrez-Rexach. Londres-NuevaYork: Routledge. 622631.

Dewaele, Jean-Marc. 2011. "Self-Reported Use and Perception of the L1 and L2 Among Maximally Proficient Bi-And Multilinguals: A Quantitative And Qualitative Investigation". International Journal of the Sociology of Language 208: 25-51.

Durán, Teresa y Nelly Ramos. 1989. "Comportamiento lingüístico en población escolar mapuche contemporánea como expresión del problema de la vitalidad de la lengua”. Actas de Lengua y Literatura Mapuche 3: 57-71.

Elder, Catherine, Tim McNamara, Hyejeong Kim, John Pill y Takanori Sato. 2017. "Interrogating the Construct of Communicative Competence in Language Assessment Contexts: What The Non-Language Specialist Can Tell Us". Language \& Communication 57: 14-21.

Espinosa, Victoria. 2003. "El español hablado por niños aymaras chilenos". Literatura y lingüistica 14: 159-171.

Espinosa, Victoria. 2009. "El aymara en la Región de Arica y Parinacota". Boletín de Filología 44, 1: Pág-39-53.

Fallas Escobar, Christian. 2016. "Challenging the Monolingual Bias in EFL Programs: Towards a Bilingual Approach to L2 Learning”. Revista de Lenguas Modernas 24: 249266.

Fishman, Joshua. 1991. Reversing language shift: Theoretical and empirical foundations of assistance to threatened languages. Vol. 76. Clevedon: Multilingual Matters.

Flores, Nelson y Jonathan Rosa. 2015. "Undoing Appropriateness: Raciolinguistic Ideologies and Language Diversity in Education." Harvard Educational Review 85, 2: 149171.

Gal, Susan. 1992. "Multiplicity and Contention Among Ideologies". Pragmatics. Quarterly Publication of the International Pragmatics Association (IPrA) 2, 3: 445-449.

Ganuza, Natalia, y Christina Hedman. 2017. "Ideology vs. Practice: Is there a Space for Trans- 
languaging in Mother Tongue Instruction?”. New Perspectives on Translanguaging and Education. Ed. BethAnne Paulsrud et al. Clevedon: Multilingual Matters. 208-226

García, Ofelia. 2009. Bilingual Education in the 21st Century: A Global Perspective. Oxford: Wiley-Blackwell.

García, Ofelia, y Rosario Torres-Guevara. 2010. "11 Monoglossic Ideologies and Language Policies in the Education of US Latinas/os". Handbook of Latinos and education: Theory, Research, and Practice. Ed. Enrique Murillo, Sofia Villenas, Ruth Trinidad Galván, Juan Sánchez Muñoz, Corinne Martínez y Margarita Machado-Casas. Nueva York: Routledge. 182-193

García, Ofelia, Nelson Flores, y Massimiliano Spotti. eds. 2016. "Language and Society: A Critical Poststructuralist Perspective". The Oxford Handbook of Language and Society. Ed. Ofelia García, Nelson Flores, y Massimiliano Spotti. Nueva York: Oxford University Press. 1-16.

Gumperz, John. 1964. "Linguistic and Social Interaction in Two Communities". American Anthropologist 66.6/2: 137-53.

Gundermann, Hans, Héctor González y Jorge Iván Vergara. 2007. "Vigencia y desplazamiento de la lengua aymara en Chile". Estudios Filológicos 42: 123-140.

Gundermann, Hans, Jaqueline Canihuan, Alejandro Clavería y Cesar Faúndez. 2008. Perfil sociolingüistico de comunidades mapuches de la Región del Biobio, Araucanía, los Rios y los Lagos. Informe de Investigación. Santiago: CONADI - UTEM.

Gundermann, Hans, Jorge Vergara y Héctor González. 2009. "El proceso de desplazamiento de la lengua aymara en Chile". Cuadernos Interculturales 7, 12: 47-77.

Heller, Monica. 2009. "14 Media, the State and Linguistic Authority". Language Ideologies and Media Discourse: Texts, Practices, Politics. Ed. Johnson, Sally y Tommaso Milani. Londres: Continuum. 277-282.

Henríquez, Marisol. 2014. "Estado del mapudungun en comunidades pewenches y lafkenches de la region del Bío-Bío: el caso de los escolares”. RLA. Revista de lingüistica teórica y aplicada 52, 2: 13-40.

Hermosilla, Julia. 1998. "Presencia de la lengua mapuche en la interpretación conversacional del niño”. Lengua y Literatura Mapuche 8: 169-177.

Hernández, Arturo y Nelly Ramos. 1978. "Rasgos del castellano hablado por escolares rurales mapuches. Estudio de un caso". RLA 16: 141-150.

Hill, Jane H. 2002. "'Expert Rhetoric's" in Advocacy for Endangered Languages: Who is Listening, and What Do They Hear?" Journal of linguistic anthropology 12.2: 119133.

Hymes, Dell. 1992. "The Concept of Communicative Competence Revisited." Thirty Years of Linguistic Evolution: Studies in Honour of René Dirven on the Occasion of his $60^{\text {th }}$ Birthday. Ed. Martin Pütz. Amsterdam: John Benjamins. 31-57.

Irvine, Judith y Susan Gal. 2000. "Language Ideology and Linguistic Differentiation". Regimes of Language: Ideologies, Polities and Identities. Ed. Paul Kroskrity. Santa Fe, NM: School of American Research Press. 35-83. 
Kirsten, Johanita. 2017. "What is in a Language: Essentialism in Macro-Sociolinguistic Research on Afrikaans". International Journal of the Sociology of Language 248: 159-195.

Kondic, Ana. 2015. Hacia un perfil sociolingüistico del Huilliche / Tsesungun de la región de Los Lagos. Fundación Aitué.

Kroskrity, Paul. 2004. "Language Ideologies". A companion to linguistic anthropology. Ed. Alessandro Duranti. Malden, MA: Blackwell. 496-517.

Kyratzis, Amy, Jennifer Reynolds y Ann-Carita Evaldsson. 2010. "Introduction: Heteroglossia and Language Ideologies in Children's peer Play Interactions”. Pragmatics 20, 4: 457-66

Lagos, Cristián. 2012. "El mapudungun en Santiago de Chile: vitalidad y representaciones sociales en los mapuches urbanos”. RLA. Revista de lingüistica teórica y aplicada 50.1: 161-184.

Lagos, Cristián. 2017. "Antropología lingüística y contacto lingüístico: ideologías lingüisticas en conflicto en contextos escolares de Pitril y Callaqui, Alto Bío Bío”. Lenguas y Literaturas Indoamericanas 19, 1:61-81

Lagos, Cristián, Cristián Oyarzo, Héctor Mariano, Daniela Molina y Felipe Hasler. 2009. "Perfil etno y sociolingüístico del mapudungún en Santiago de Chile". Lenguas Modernas 34: 117-137.

Lagos, Cristián, Marco Espinoza y Darío Rojas. 2013. "Mapudungun According to its Speakers: Mapuche Intellectuals and the Influence of Standard Language Ideology". Current Issues in Language Planning 14: 403-418.

Makihara, Miki. 2005. "Rapa Nui Ways of Speaking Spanish: Language Shift and Socialization on Easter Island". Language in Society 34, 5: 727-762.

Milroy, James. 2001. "Language Ideologies and the Consequences of Standardization”. Journal of sociolinguistics 5, 4: 530-555.

MINEDUC. 2017. Programa de estudio octavo año básico. Sector lengua indígena mapuzugun.

McKinney, Carolyn., Hannah Carrim, Alex Marshall, y Laura Layton. 2015. "What counts as language in South African schooling? Monoglossic Ideologies and Children's Participation". AILA Review 28: 103-126.

McKinney, Carolyn. 2016. Language and Power in Postcolonial Schooling: Ideologies in Practice. Nueva York: Routledge

Moore, Robert, Sari Pietikäinen y Jan Blommaert. 2010. "Counting the losses: Numbers as the language of language endangerment". Sociolinguistic Studies 4.1: 1-26.

Ojeda, Patricia, y Javier Âlvarez. 2014. "Elementos para la construcción social del chedungun a partir del discurso en torno a la lengua de hablantes bilingües de la VIII Región”. Boletín de filología 49, 2: 161-185.

Olate, Aldo. 2012. Y después, lo estaban mirando a la rana. La competencia lingüístico-comunicativa del castellano en escolares de una comunidad mapuche/castellano. Tesis para optar al grado de Doctor en Lingüística, Universidad de Concepción.

Olate, Aldo. 2017a. "Contacto lingüístico mapuzugun/castellano. Aspectos históricos, sociales y lingüísticos. Revisión bibliográfica y propuesta de análisis desde la dimensión 
morfosintáctica y tipológica”. ONOMÁZEIN 36: 122-158.

Olate, Aldo. 2017b. "Más allá del estado de vida de las lenguas... elementos para el diagnóstico sociolingüístico de la dinámica interactiva del contacto mapuzugun/castellano". Alpha (Osorno) 45: 255-272.

Olate, Aldo, Fernando Wittig, y Felipe Hasler. 2014. "Análisis tipológico-funcional de un rasgo del español de contacto mapuche/castellano". ONOMÁZEIN 30: 170-189.

Olate, Aldo, Cesar Cisternas, Fernando Wittig, y Jaime Flores. 2017. "Los misioneros capuchinos bávaros y sus ideologías lingüísticas sobre la lengua mapuche”. Nueva revista del Pacífico 67: 130-156.

Paffey, Darren. 2012. Language Ideologies and the Globalization of 'Standard' Spanish. Londres: Bloomsbury.

Pauwels, Anne. 2016. Language Maintenance and Shift. Cambridge: Cambridge University Press.

Piller, Ingrid. 2015. "Language Ideologies". The International Encyclopedia of Language and Social Interaction. Vol. 1. Ed. Karen Tracy, Cornelia Ilie y Todd Sandel. John Wiley \& Sons. 1-10.

Poza, Luis E. 2016. "Pure Spelling and Grammar": Conceptualizations of Language and the Marginalization of Emergent Bilinguals." Penn GSE Perspectives on Urban Education 13.1: 20-41.

Pozo, Gabriel. 2014. “¿Cómo descolonizar el saber? El problema del concepto de interculturalidad". Revista Polis 38: 1-13.

Relmuan, María Angélica. 2005. El mapuche, el aula y la formación docente. Vol. 7. La Paz: Plural Editores.

Ricento, Thomas. ed. 2009. An Introduction to Language Policy: Theory and method. John Wiley \& Sons.

Rojas, Darío. 2015. "Ideologías y actitudes lingüísticas en el Chile del siglo XIX: los reparos de Andrés Bello a las Correcciones lexigráficas de Valentín Gormaz". Lexis 39, 1: 163-181.

Rojas, Darío, Cristián Lagos y Marco Espinoza. 2016. “Ideologías lingüísticas acerca del mapudungun en la urbe chilena: el saber tradicional y su aplicación a la revitalización lingüística”. Chungará 47: 1-11.

Romaine, Suzanne. 2000. Language in Society: An Introduction to Sociolinguistics. Oxford University Press.

Rosa, Jonathan, y Christa Burdick. 2016. "Language Ideologies". The Oxford Handbook of Language and Society. Ed. Ofelía García, Nelson Flores, y Massimiliano Spotti. Nueva York: Oxford University Press. 103-123.

Rumsey, Alan. 1990. "Wording, Meaning, and Linguistic Ideology". American Anthropologist 92, 2: 346-361.

Salö, Linus. 2018. "Seeing The Point From Which You See What You See: An Essay on Epistemic Reflexivity in Language Research." Multilingual Margins: A journal of Multilingualism From The Periphery 5, 1:24-39. 
Schieffelin, Bambi, Kathryn Woolard y Paul Kroskrity. eds. 1998. Language Ideologies: Practice and Theory. Vol. 16. Nueva York: Oxford University Press.

Sepúlveda, Gastón. 1984. "Vitalidad etnolingüística de la lengua araucana”. CUHSO. Cultura-Hombre-Sociedad 1.1: 223-238.

Silverstein, Michael. 1979. "Language Structure and Linguistic Ideology". The Elements: A Parasession on Linguistic Units and Levels. Ed. Paul Clyne, William Hanks, y Carol Hofbauer. Chicago: Chicago Linguistic Society. 219-259.

Teillier, Fernando. 2013. "Vitalidad lingüística del mapudungun en Chile y epistemología del Hablante". Revista de Lingüistica Teórica y Aplicada 51, 1: 53-70.

Teillier, Fernando, Gabriel Llanquinao y Gastón Salamanca. 2016. "De qué hablamos cuando hablamos de etnolingüística: bases teórico-metodológicas para un trabajo con el mapunzugun”. RLA. Revista de lingüistica teórica y aplicada 54.2: 137-161.

Valdés, Guadalupe. 2017. "From language maintenance and intergenerational transmission to language survivance: will "heritage language" education help or hinder?." International Journal of the Sociology of Language 243: 67-95.

Vogel, Sara, y Ofelía García. 2017. "Translanguaging". Oxford Research Encyclopedia of Education. Eds. George Noblit y Luis Moll. Oxford: Oxford University Press.

Wardhaugh, Ronald y Janet Fuller. 2015. An Introduction to Sociolinguistics (7th ed.). USA: Wiley-Blackwell.

Wittig, Fernando y Aldo Olate. 2016. "El mapuzugun en La Araucanía. Apuntes en torno al desfase entre la politización de la lengua y la heterogeneidad sociolingüística local". UniverSOS 13: 119-134.

Woolard, Kathryn. 1998. "Introduction: Language Ideology as a Field of Inquiry". Language Ideologies. Practice and Theory. Ed. Bambi Schieffelin, Kathryn Woolard y Paul Kroskrity. New York and Oxford: Oxford University Press. 3-47

Zavala, Virginia. 2016. "Ideologías sobre el quechua desde el poder: una aproximación discursiva”. Signo y Seña 29: 207-234.

Zemelman, Hugo. 2001. "Pensar teórico y pensar epistémico: los retos de las ciencias sociales latinoamericanas". Conferencia Magistral, Universidad de la Ciudad de México. Vol. 10.

Zúñiga, Fernando. 2007. "Mapuduguwelaymi am ¿acaso ya no hablas mapudungun? Acerca del estado actual de la lengua mapuche". Estudios Públicos 105: 9-24.

Zúñiga, Fernando, y Aldo Olate. 2017. "El estado de la lengua mapuche, diez años después" El pueblo mapuche en el siglo XXI: propuestas para un nuevo entendimiento entre culturas en Chile. Ed. Isabel Aninat, Verónica Figueroa, y Ricardo González. Santiago de Chile: Centro de Estudios Públicos. 343-374. 
